\title{
Unexpected COVID-19 Infection during Immediate Postoperative Period: A Call for Concern
}

Minati Choudhury ${ }^{1}$ Milind P. Hote ${ }^{1}$ Poonam Malhotra Kapoor ${ }^{1}$
Address for correspondence Minati Choudhury, MD, PGDip, Department of Cardiac Anesthesiology, Cardiothoracic Sciences Centre, All India Institute of Medical Sciences, Ansari Nagar, New Delhi 110029, India (e-mail: minati.2002@gmail.com).

The total number of Coronavirus disease 2019 (COVID-2019) cases has reached a shocking 8 million, and the number continues to increase as countries across the world are easing lockdown restrictions. This pandemic has had a significant impact on the practice of cardiothoracic surgery, owing to the increasing burden on clinical resources and concern for nosocomial spread.

At one end, the healthcare system is trying to cope with this pandemic, while on the other end, the major ongoing concern is about the active spread of the disease from individuals who are infected but without symptoms. ${ }^{1}$

Perioperative screening has been started in all centers for patients undergoing elective surgery. However, in emergent and urgent situations, for example, acute coronary syndrome, acute cardiac tamponade, acute aortic dissection, acute valvular endocarditis or patients with recurrent "tet" spell, they may need to be taken to the operating room without being tested for COVID-19 infection. Another major issue occurs when the postoperative patient suddenly turns positive on postoperative day one of surgery with varied symptoms pertinent to COVID-19 after an initial negative test. These issues have a direct implication for the safety of healthcare workers and for the success of the effort to halt the pandemic. The false negative results are important because of various reasons. Patients with these results may be ignored and the general measures designed to reduce the transmission of the virus to others is relaxed. In case of clinicians, they may be sent to frontline care and inadvertently transmit the virus to patients and colleagues, further straining the already precarious ability of the healthcare system to respond to the pandemic. A postoperative cardiac surgical patient with overt COVID-19 infection may have a complicated postoperative course and need additional care pertinent to the symptoms related to this pandemic. One of the reported cases was diagnosed to have COVID19 pneumonia after testing of the bronchopulmonary

published online

November 2, 2020
DOI https://doi.org/ 10.1055/s-0040-1721185 ISSN 2457-0206. lavage. ${ }^{2}$ Coagulation abnormality and thrombosis was noted in another patient. ${ }^{3}$ When a postoperative patient tests positive from COVID-19, the management starts immediate shifting of the patient to the COVID-19 care unit of the hospital or to an isolation area. Monitoring and screening of the remaining patients in the unit as well as thromboembolism prophylaxis can avoid unanticipated problems among this population. ${ }^{4}$

The doctors, healthcare workers, ancillary workers who are involved in the management of the patient in the operating room (OR), as well as the postoperative intensive care unit need to be urgently identified and quarantined after taking advice from the infectious disease department. They may be considered at elevated risk of COVID-19 infection even without much symptoms or being asymptomatic and need to undergo the COVID-19 diagnostic test within the recommended time. The prophylactic regimen, for example, vitamin $\mathrm{D}$, vitamin $\mathrm{C}$, famotidine and hydroxychloroquine need to be administered according to the guidelines. ${ }^{5}$ These viruses live for 72 hours on metal and plastic. The virions after getting sucked by the exhaust fan will blow into the OR as aerosol through the ventilation system. Hence, it is essential to fumigate the OR to eradicate this threat. Following strict decontamination of the OR, the hospital's infectious disease department may be requested to check the presence of virus in the OR. Careful cleaning and decontamination of the usable material is warranted.

In conclusion, COVID-19 diagnosis in the immediate postoperative period is more alarming and challenging. The clinical presentation may be similar to that of other septic, cardiovascular, pulmonary, thromboembolic, or gastrointestinal postsurgical complications. In addition, the postoperative period may witness an increased risk of severe manifestation of COVID-19, which needs aggressive care and strict monitoring. The prognosis is usually poor because of several factors like effect of general anesthesia, blood loss/ 
transfusion, and systemic inflammatory response associated with cardiopulmonary bypass (CPB). These things need to be explained to the attendants. In the era of uncertainty, we must be aware of unexpected COVID-19 diagnosis in the immediate postoperative period.

\section{Conflicts of Interest}

None declared.

\section{References}

1 Li RPaiSChenB, et al. Substantial under documented infection facilitates the rapid dissemination of novel corona virus (SARS-CoV2). Science 2020;(e-pub ahead of print). Doi:10.1126/science.abb3221
2 Rescigno G, Firstenberg M, Rudez I, Uddin M, Nagarajan K, Nikolaidis N. A case of postoperative COVID-19 infection after cardiac surgery: lessons learned. Heart Surg Forum 2020; 23(2):E231-E233

3 Levi M, Thachil J, Iba T, Levy JH. Coagulation abnormalities and thrombosis in patients with COVID-19. Lancet Haematol 2020;7(6):e438-e440

4 Iba T, Levy JH, Levi M, Connors JM, Thachil J. Coagulopathy of coronavirus disease 2019. CritCare Med 2020;48(9):1358-1364

5 Burke RM, Midgley CM, Dratch A, et al. Active monitoring of persons exposed to patients with confirmed COVID-19- United States, January-February 2020. MMWR Morb Mortal Wkly Rep 2020;69(9):245-246 